\title{
Remote monitoring of patients with cardiac implantable electronic devices: a Southeast Asian, single-centre pilot study
}

Paul Chun Yih Lim ${ }^{1}$ MBBS, MRCP, Audry Shan Yin Lee ${ }^{1}$, MBBChir, MRCP, Kelvin Chi Ming Chua ${ }^{1}$ MBBs, MRCP, Eric Tien Siang Lim ${ }^{1}$, MBBS, MRCP, Daniel Thuan Tee Chong ${ }^{1}$, MBBS, MRCP, Boon Yew Tan ${ }^{1}$, MBChB, MRCP, Kah Leng $\underline{\mathrm{HO}}^{1}$, MBBS, MRCP, Wee Siong ${\underline{T e 0^{1}}}^{1}$ MBBS, MRCP, Chi Keong $\underline{\text { Ching }}{ }^{1}$, MBBS, MRCP

INTRODUCTION Remote monitoring of cardiac implantable electronic devices (CIED) has been shown to improve patient safety and reduce in-office visits. We report our experience with remote monitoring via the Medtronic CareLink ${ }^{\circledR}$ network. METHODS Patients were followed up for six months with scheduled monthly remote monitoring transmissions in addition to routine in-office checks. The efficacy of remote monitoring was evaluated by recording compliance to transmissions, number of device alerts requiring intervention and time from transmission to review. Questionnaires were administered to evaluate the experiences of patients, physicians and medical technicians.

RESULTS A total of 57 patients were enrolled; 16 (28.1\%) had permanent pacemakers, 34 (59.6\%) had implantable cardioverter defibrillators and $7(12.3 \%)$ had cardiac resynchronisation therapy defibrillators. Overall, of 334 remote transmissions scheduled, $73.7 \%$ were on time, $14.5 \%$ were overdue and $11.8 \%$ were missed. $84.6 \%$ of wireless transmissions were on time, compared to $53.8 \%$ of non-wireless transmissions. Among all transmissions, $4.4 \%$ contained alerts for which physicians were informed and only $1.8 \%$ required intervention. $98.6 \%$ of remote transmissions were reviewed by the second working day. $73.2 \%$ of patients preferred remote monitoring. Physicians agreed that remote transmissions provided information equivalent to in-office checks $97.1 \%$ of the time. $77.8 \%$ of medical technicians felt that remote monitoring would help the hospital improve patient management. No adverse events were reported. CONCLUSION Remote monitoring of CIED is safe and feasible. It has possible benefits to patient safety through earlier detection of arrhythmias or device malfunction, permitting earlier intervention. Wireless remote monitoring, in particular, may improve compliance to device monitoring. Patients may prefer remote monitoring due to possible improvements in quality of life.

Keywords: cardiac implantable electronic devices, remote monitoring

\section{INTRODUCTION}

Advances in technology have allowed the remote transmission of data from cardiac implantable electronic devices (CIED), i.e. permanent pacemakers (PPMs), implantable cardioverter defibrillators (ICDs) and cardiac resynchronisation therapy defibrillators (CRT-Ds). Remote access to device diagnostics has been shown to improve patient safety in comparison to ambulatory conventional monitoring due to earlier detection of arrhythmias or device malfunction. ${ }^{(1-4)}$ It has also significantly reduced the number of in-office visits, workload of healthcare providers and healthcare costs to patients. ${ }^{(5,6)}$ In addition, patient compliance to scheduled remote monitoring evaluation is superior compared to in-office checks. ${ }^{(5)}$ Home monitoring is relatively new in Southeast Asia, ${ }^{(1,7)}$ with pickup rates increasing over recent years.

We report our experience with home monitoring among patients with Medtronic (Medtronic Inc, Minneapolis, MN, USA) devices in this pilot study, which aimed to describe patient compliance to scheduled remote monitoring transmissions as well as physician, patient and healthcare workers' experiences with remote monitoring. In Singapore, distance from CIED monitoring centres is much less of a barrier in comparison to larger countries. Hence, this study was also an examination of patients' opinions regarding remote monitoring in a situation where geographical distance is less of a barrier toward office device monitoring.

\section{METHODS}

We conducted a prospective study from June 2012 to December 2013 involving patients who were implanted with Medtronic CIED for standard indications. Patients from the National Heart Centre Singapore were recruited for remote monitoring via the Medtronic CareLink ${ }^{\circledR}$ network. Selection criteria required only that patients or their caretakers were able to understand and willing to carry out the instructions given for remote monitoring. Patients were followed up for six months with scheduled monthly remote monitoring transmissions, in addition to routine in-office checks during specialist outpatient clinic reviews. Additional remote monitoring checks could be scheduled at the attending physician's discretion.

Patients enrolled in the study were equipped with a patient monitor with a telephone or Global System for Mobile communications (GSM) network connection. Non-wireless and wireless home monitors were used. Device interrogation with

${ }^{1}$ Department of Cardiology, National Heart Centre Singapore, Singapore

Correspondence: A/Prof Ching Chi Keong, Senior Consultant, Department of Cardiology, National Heart Centre Singapore, 5 Hospital Drive, Singapore 169609. ching.chi.keong@nhcs.com.sg 
the non-wireless home monitor was performed by placing an interrogation antenna over the implanted device. Wireless home monitors automatically interrogated the devices periodically for scheduled transmissions and could be triggered by device or clinical alerts. Data was transmitted securely to the CareLink network and results were accessed via the Internet-accessible CareLink website. In our centre, the medical technician is the first-line recipient of remote transmissions. The medical technician vets the data from the remote transmissions to detect any alerts that require early communication to the electrophysiologist. Significant alerts included sustained arrhythmias, abrupt increases in lead impedance or thresholds, and battery replacement indicators based on predefined protocols.

Enrolled patients were followed up to review the efficacy and safety of the remote monitoring. Outcomes measured were compliance to scheduled remote transmissions (i.e. if they were on time, overdue [received 1-5 days after the scheduled date] or missed [received $>5$ days after the scheduled date]); time from transmission to review; occurrence of unscheduled transmissions arising from device alerts; and number of alerts that required further intervention. Questionnaires were administered to physicians during the course of the six months of follow-up, seeking physicians' opinions on whether remote monitoring yielded equivalent information as compared to inoffice checks, and preferred frequency for device checks and follow-up visits.

Patients answered a questionnaire to determine their employment status, need to be accompanied and amount of time required for device checks, taking into account travel and waiting times. They were also surveyed on their preferences regarding remote monitoring as compared to in-office checks and if remote monitoring had a positive effect on their peace of mind. This questionnaire was completed at the end of the six-month remote monitoring experience.

Lastly, a questionnaire was also completed by medical technicians involved in the remote monitoring process. The questionnaire surveyed their opinions regarding ease of use of the remote monitoring system; whether it provided an equivalent service to patients compared to in-office checks; and if it could improve clinical and workflow efficacy.

\section{RESULTS}

A total of 57 patients were recruited over a period of eight months, consisting of 16 (28.1\%) patients with PPMs, 34 (59.6\%) patients with ICDs and 7 (12.3\%) patients with CRT-Ds. Patients who received a PPM had the following indications: sinus node dysfunction (62.5\%), atrioventricular node dysfunction (31.3\%), or both $(6.3 \%)$. The ICD and CRT-D cohorts comprised mainly of patients with primary prevention indications for ischaemic and non-ischaemic cardiomyopathy. Mean left ventricular ejection fraction was $35.5 \% \pm 16.5 \%$ in the ICD cohort and $19.9 \% \pm 4.8 \%$ in the CRT-D cohort. Population baseline characteristics are shown in Fig. 1 and Table I.

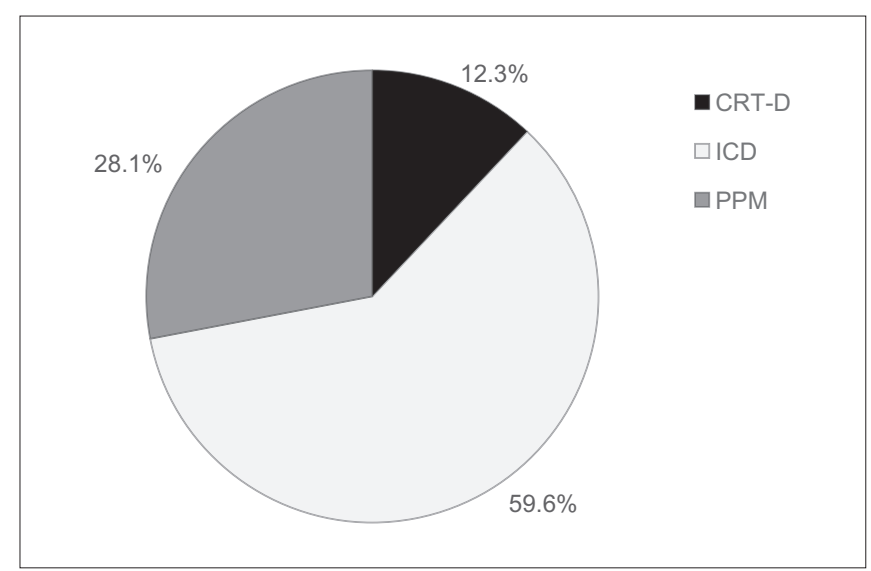

Fig. 1 Pie chart shows patient enrolment by cardiac implantable electronic device type. CRT-D: cardiac resynchronisation therapy defibrillator; ICD: implantable cardioverter defibrillator; PPM: permanent pacemaker

Table I. Characteristics of patients from each cardiac implantable electronic device group $(n=57)$.

\begin{tabular}{|c|c|c|c|}
\hline \multirow[t]{2}{*}{ Characteristic } & \multicolumn{3}{|c|}{ No. (\%) } \\
\hline & $\begin{array}{c}\text { PPM } \\
(n=16)\end{array}$ & $\begin{array}{c}\text { ICD } \\
(n=34)\end{array}$ & $\begin{array}{l}\text { CRT-D } \\
(n=7)\end{array}$ \\
\hline Age* $(y r)$ & $65.8 \pm 9.2$ & $57.9 \pm 15.8$ & $60.4 \pm 7.1$ \\
\hline LVEF* (\%) & $60.5 \pm 5.9$ & $35.5 \pm 16.5$ & $19.9 \pm 4.8$ \\
\hline \multicolumn{4}{|l|}{ Comorbidity $^{+}$} \\
\hline $\begin{array}{l}\text { Ischaemic heart } \\
\text { disease }\end{array}$ & $2(12.5)$ & $22(64.7)$ & $3(42.9)$ \\
\hline Atrial fibrillation & $7(43.8)$ & $7(20.6)$ & 0 \\
\hline Diabetes mellitus & $2(12.5)$ & $8(23.5)$ & $4(57.1)$ \\
\hline Hypertension & $14(87.5)$ & $24(70.6)$ & $4(57.1)$ \\
\hline Hyperlipidaemia & $10(62.5)$ & $24(70.6)$ & $5(71.4)$ \\
\hline Chronic kidney disease & $1(6.3)$ & $4(11.8)$ & $2(28.6)$ \\
\hline \multicolumn{4}{|l|}{ Pacemaker cohort } \\
\hline SND & $10(62.5)$ & - & - \\
\hline AVN dysfunction & $5(31.3)$ & - & - \\
\hline $\begin{array}{l}\text { SND and AVN } \\
\text { dysfunction }\end{array}$ & $1(6.3)$ & - & - \\
\hline \multicolumn{4}{|l|}{ ICD/CRT-D cohort ${ }^{\dagger}$} \\
\hline Primary prevention & - & $23(67.6)$ & $1(14.3)$ \\
\hline Secondary prevention & - & $11(32.4)$ & $6(85.7)$ \\
\hline $\begin{array}{l}\text { Ischaemic } \\
\text { cardiomyopathy }\end{array}$ & - & $19(55.9)$ & $3(42.9)$ \\
\hline $\begin{array}{l}\text { Dilated } \\
\text { cardiomyopathy }\end{array}$ & - & $8(23.5)$ & $4(57.1)$ \\
\hline $\begin{array}{l}\text { Hypertrophic } \\
\text { cardiomyopathy }\end{array}$ & - & $2(5.9)$ & 0 \\
\hline Sarcoidosis & - & $1(2.9)$ & 0 \\
\hline Long QT syndrome & - & $1(2.9)$ & 0 \\
\hline VT/VF collapse & - & $4(11.8)$ & 0 \\
\hline \multicolumn{4}{|l|}{ Home monitor type } \\
\hline Wireless & 0 & $32(94.1)$ & $5(71.4)$ \\
\hline Non-wireless & $16(100.0)$ & $2(5.9)$ & $2(28.6)$ \\
\hline
\end{tabular}

* Data presented as mean \pm standard deviation. †Patients could have $>1$ indication. AVN: atrioventicular node; CRT-D: cardiac resynchronisation therapy defibrillator; ICD: implantable cardioverter defibrillator; LVEF: left ventricular ejection fraction; PPM: permanent pacemaker; SND: sinus node dysfunction; VF: ventricular fibrillation; VT: ventricular tachycardia 
A total of 334 remote transmissions were scheduled, comprising 215 wireless and 119 non-wireless transmissions. Among wireless transmissions, $84.6 \%$ were on time, $5.1 \%$ were overdue and $10.3 \%$ were missed. Among non-wireless transmissions, 53.8\% were on time, $31.6 \%$ were overdue and $14.5 \%$ were missed. Overall, among all remote transmissions, $73.7 \%$ were on time, $14.5 \%$ were overdue and $11.8 \%$ were missed.

There were a total of 15 alerts arising from 340 transmissions, all of which were from the ICD/CRT-D cohorts. Nine alerts were noted during scheduled remote transmissions, of which $3(33.3 \%)$ transmissions required intervention; one was an Optivol ${ }^{\circledR}$ alert and the rest were arrhythmia-triggered, which resulted in early follow-up. The six unscheduled device alert transmissions all arose from ICD arrhythmia episodes. 3 (50.0\%) of the six unscheduled transmissions required intervention: one patient received an appropriate shock and had multiple episodes of ventricular tachycardia that prompted an admission to hospital, and the remaining two alerts resulted in earlier follow-ups for ventricular tachycardia, resulting in device therapy. Overall, $4.4 \%$ of transmissions had alerts and $1.8 \%$ of all transmissions required intervention. $98.6 \%$ of remote transmissions were reviewed by the second working day, with $71.6 \%$ being reviewed on the same day as transmission, $22.2 \%$ by the next day and an additional $4.8 \%$ on the second working day.

The patient questionnaire, administered to 41 patients in the cohort, revealed that $73.2 \%$ of patients preferred to send device information from home instead of undergoing an in-office check. Having a remote monitor for device monitoring accorded additional peace of mind to $85.4 \%$ of patients (Fig. 2). All patients agreed that setup and utilisation of the remote monitor was either easy or very easy. $90.2 \%$ of patients were keen to continue remote monitoring after cessation of the trial, citing convenience as the most common reason, followed by time-saving benefits. $43.9 \%$ of patients were actively employed and required leave from work to attend in-office device checks. $63.4 \%$ of patients claimed that an in-office check would require an average of 1-3 hours of their time, taking into account transport and waiting times, while $19.5 \%$ of patients said they spent more than three hours. $58.5 \%$ of patients were accompanied by a caregiver for these checks (Table II).

The physician questionnaire was administered to managing physicians of 36 patients after three months of remote monitoring. Physicians felt that $97.1 \%$ of remote transmissions accorded them equivalent information compared to in-office checks. The mean preferred frequency for in-clinic follow-up visits was every 8.5 months and the average preferred interval for device checks was 3.3 months.

The medical technician questionnaire, which was completed by nine medical technicians, showed that $77.8 \%$ of them felt that remote monitoring would help the hospital improve patient management and $66.7 \%$ felt it would enhance efficiency in the long run; $77.8 \%$ said the level of assistance provided to patients through remote follow-up was equivalent to that provided during in-office device checks.

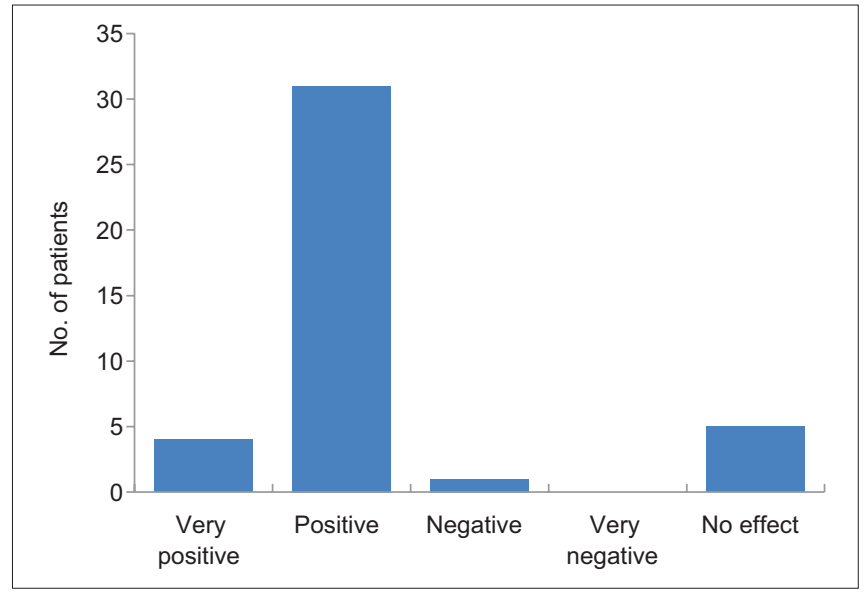

Fig. 2 Bar chart shows patients' responses to whether remote monitoring gave them peace of mind.

Table II. Patient questionnaire results $(n=41)$.

\begin{tabular}{lc}
\hline Response & No. (\%) \\
\hline Duration of visit (hr) & $7(17.1)$ \\
$<1$ & $26(63.4)$ \\
$1-3$ & $8(19.5)$ \\
$>3$ & \\
Accompanied by caregiver during visit & $16(39.0)$ \\
Always & $17(41.5)$ \\
Never & $8(19.5)$ \\
Sometimes & \\
Employed & $18(43.9)$ \\
Yes & $23(56.1)$ \\
No & \\
\hline
\end{tabular}

\section{DISCUSSION}

In our pilot study, there was good compliance to scheduled transmissions, which was more evident in the wireless monitoring cohort. The number of device alerts that required intervention was low and remote transmissions were reviewed promptly. Patients, physicians and medical technicians largely had a favourable opinion of remote monitoring.

In an ageing population, CIED implantation rates are likely to increase. ${ }^{(7)}$ A large proportion of these patients are at high risk and require close attention. ${ }^{(5)}$ Prior to remote monitoring, the standard of care for CIED patients would require in-office device checks at least every 3-6 months, with certain groups of patients needing even more frequent follow-up. ${ }^{(8)}$ The advent of remote monitoring has allowed clinicians remote access to device diagnostic information relating to device integrity and function; both atrial and ventricular arrhythmias; arrhythmia therapy; and, in some devices, impending fluid overload. ${ }^{(9,10)}$ Data is downloaded from the device via automatic wireless interrogation through a built-in device micro antenna or by wand activation. Information is transmitted through land telephone lines or cellular GSM networks to the manufacturers' centre and subsequently disseminated to relevant medical personnel. The physician can customise the type of alerts that will trigger a transmission and choose to be notified through fax, email, short 
message or pager. Device reprogramming, however, cannot be done remotely, in order to avoid unauthorised control of device function. ${ }^{(11,12)}$ Utilisation of remote monitoring in the Southeast Asian region is relatively low compared to other regions. The reasons for this vary widely between countries, but a significant reason is the increased cost of the home monitoring setup in comparison to conventional devices. ${ }^{(7)}$ Singapore was one of the first in the region to employ home monitoring.

In terms of safety, current data shows that the majority of routine in-office device checks do not require intervention, as remote monitoring accurately excludes device malfunction or arrhythmias in patients. ${ }^{(13)}$ Our data appears to reflect this trend, with a low number of alerts that required intervention (i.e. 1.8\% of all transmissions). All alerts during our study were from the ICD cohort, with 14 (93.3\%) of the 15 alerts being arrhythmiarelated. Remote monitoring permits an earlier reaction to events requiring physician intervention in comparison to regular in-office device checks, with $98.6 \%$ of transmissions being reviewed within two working days in our cohort. ${ }^{(3,4,6,14-21)}$ Early detection allows prompt management of arrhythmias, early management of device malfunction and device reprogramming to reduce inappropriate defibrillator shocks. ${ }^{(18,22,23)}$

Compliance to remote monitoring was good, with an overall missed transmission rate of $11.8 \%$. This takes into account the more intensive schedule of monthly checks during this pilot study in comparison to common practice worldwide. Unsurprisingly, the wireless monitor cohort had lower missed and overdue transmission rates compared to the non-wireless group. Observational data has shown potentially higher survival rates for ICD/CRT-D patients on remote monitoring. ${ }^{(24)}$ Surveillance for fluid status has been reported to substantially reduce heart failure-related hospitalisations and death. ${ }^{(25)}$ The use of remote monitoring could reduce the number of office visits, lower time consumption for physicians and healthcare workers, and provide more efficient healthcare delivery without compromising, and possibly improving, care standards. ${ }^{(3,4,6,26-30)}$

Singapore is geographically the second smallest country in Asia and amongst the 25 smallest countries in the world. With a high population density (estimated as $7,615 / \mathrm{km}^{2}$ ), geographical distance is far less of a barrier to in-office CIED monitoring than in larger countries. ${ }^{(34)}$ Despite this, $71.9 \%$ of our patient cohort still preferred remote monitoring to inoffice checks to complement regular clinic follow-up visits, citing a reduced need for time-off from work or for caregivers to accompany them to the clinic. Of the patients surveyed, $58.5 \%$ were accompanied by caregivers on all or some of their in-clinic visits and $43.9 \%$ were employed. According to our questionnaire results, remote transmission saved a median of three hours per visit for both patients and any accompanying caregivers. The time and cost savings for this cohort of patients and their families is likely to be significant, although they have to be weighed against the initial cost of setting up the home monitoring. Remote monitoring was thus able to benefit not only the patient but also their families, regardless of the ease of access and relatively closer proximity to a CIED monitoring centre in Singapore.

Initial concerns regarding the loss of face-to-face encounters with medical staff were not observed in our cohort of remote monitoring patients. Their overall satisfaction was consistent with high satisfaction rates worldwide from enhancement of patients' quality of life. ${ }^{(6,11,31-33)}$ This preference for remote monitoring, regardless of geographic proximity, could perhaps be extrapolated to countries where there are doubts as to whether implementation of remote monitoring would benefit patients living near a monitoring centre. It may also be applied to some city-dwelling patients who are in close proximity to a monitoring centre but are hampered by mobility problems, work schedules or poor traffic conditions.

Based on the questionnaires administered, physicians found that $97.1 \%$ of the time, remote monitoring delivered the same amount of information compared to an in-office check, and medical technicians felt that the amount of assistance they could give to the patient via remote monitoring was equivalent to an in-office check $77.8 \%$ of the time. The same proportion of medical technicians felt that remote monitoring could improve patient management.

Despite their benefits, remote monitoring systems generate a plethora of data that entails a considerable need for proper information filtering. ${ }^{(35)} 98.2 \%$ of the transmissions in our study did not contain alerts that required intervention. Considering our increased frequency of routine transmissions on a monthly basis for the purposes of this feasibility study, the proportion of alerts requiring intervention is likely to be higher in a non-pilot study setting. Of alerts that were transmitted on an unscheduled basis, only $33.3 \%$ required intervention, illustrating that remote monitoring has the potential of generating large amounts of data that needs to be reviewed. At our institution, our medical technicians serve as first-line recipients of the remote transmissions. They are also able to review online medical data such as medication lists to aid our electrophysiologists in the management process. This institutional process reduced the amount of non-essential data being relayed to the electrophysiologist. Feedback from our physician survey was generally positive. However, in the clinical setting, alert settings nevertheless have to be tailored to individual patient profiles to ensure efficiency of data management.

As this study was designed to be a pilot study, its limitations included the small cohort of patients without a control population. While the selection process did not deliberately include patients of particular age, language or socioeconomic criteria, the mean age of the overall cohort was relatively low. This could reflect selection bias, as the nature of the study necessitated that participants or their caretakers were able to understand and carry out the instructions given for remote monitoring. However, the relatively young age of the participants potentially reflect a real-life clinical scenario in which younger patients may be less technology-averse and keener to adopt remote monitoring. Testing this hypothesis requires further evaluation of patients' opinions regarding 
remote monitoring. Future evaluation may also be able to detect associations between uptake of remote monitoring devices and socioeconomic status. In addition, the number of alerts and transmissions requiring intervention were low, which may be attributed to the short duration of the study and the relative newness of all the devices, resulting in a low number of device/ lead malfunction alerts.

In conclusion, our pilot study showed that remote monitoring of patients with CIED is safe and feasible with a high rate of adherence to transmission schedules. There was a low incidence of device alerts that required intervention and transmitted reports were comparable to in-office reports. Patients, physicians and medical technicians' experiences with remote monitoring were favourable. Remote monitoring of patients with CIED could provide earlier detection of arrhythmia events or device malfunction, thereby improving patient outcomes through appropriate early intervention, significantly improving quality of life for patients and their families, and positively impacting healthcare resource utilisation through proper management of transmission alert settings.

\section{ACKNOWLEDGEMENT}

We would like to acknowledge Ms Rony Weisman, Medtronic Inc, for her assistance with this study.

\section{REFERENCES}

1. Wang H, Hua W, Ding LG, et al. Home monitoring system improves the detection of ventricular arrhythmia and inappropriate shock. Chin Med J (Engl) 2012; 125:3421-4

2. Freedberg NA, Feldman A. Remote monitoring of patients with Implantable Cardioverter Defibrillators (ICD): a cute gimmick or an essential tool for clinical excellence? J Cardiovasc Electrophysiol 2014 25:771-3.

3. Varma N, Epstein AE, Irimpen A, Schweikert R, Love C; TRUST Investigators. Efficacy and safety of automatic remote monitoring for implantable cardioverter-defibrillator follow-up: the Lumos-T Safely Reduces Routine Office Device Follow-Up (TRUST) trial. Circulation 2010 ; 122:325-32.

4. Crossley $\mathrm{GH}$, Boyle A, Vitense $\mathrm{H}$, Chang $\mathrm{Y}$, Mead $\mathrm{RH}$; CONNECT Investigators. The CONNECT (Clinical Evaluation of Remote Notification to Reduce Time to Clinical Decision) trial: the value of wireless remote monitoring with automatic clinician alerts. J Am Coll Cardiol 2011; 57:1181-9.

5. Varma N, Michalski J, Stambler B, Pavri BB; TRUST Investigators. Superiority of automatic remote monitoring compared with in-person evaluation for scheduled ICD follow-up in the TRUST trial - testing execution of the recommendations. Eur Heart J 2014; 35:1345-52.

6. Hindricks G, Elsner C, Piorkowski C, et al. Quarterly vs. yearly clinical follow-up of remotely monitored recipients of prophylactic implantable cardioverter-defibrillators: results of the REFORM trial. Eur Heart J 2014 35:98-105.

7. Lau CP, Zhang S. Remote monitoring of cardiac implantable devices in the Asia-Pacific. Europace 2013; 15 Suppl 1:i65-i68.

8. Epstein AE, Dimarco JP, Ellenbogen KA, et al; American College of Cardiology; American Heart Association Task Force on Practice Guidelines; American Association for Thoracic Surgery; Society of Thoracic Surgeons ACC/AHA/HRS 2008 Guidelines for device-based therapy of cardiac rhythm abnormalities. Heart Rhythm 2008; 5:e1-62.

9. Nielsen JC, Kottkamp H, Zabel M, et al. Automatic home monitoring of implantable cardioverter defibrillators. Europace 2008; 10:729-35.

10. Burri H, Senouf D. Remote monitoring and follow-up of pacemakers and implantable cardioverter defibrillators. Europace 2009; 11:701-9.

11. Dubner S, Auricchio A, Steinberg JS, et al. ISHNE/EHRA expert consensus on remote monitoring of cardiovascular implantable electronic devices (CIEDs). Europace 2012; 14:278-93.

12. Sticherling C, Kuhne M, Schaer B, Altmann D, Osswald S. Remote monitoring of cardiovascular implantable electronic devices: prerequisite or luxury? Swiss Med Wkly 2009; 139:596-601.

13. Heidbüchel $H$, Lioen $P$, Foulon $S$, et al. Potential role of remote monitoring for scheduled and unscheduled evaluations of patients with an implantable defibrillator. Europace 2008; 10:351-7.

14. Schoenfeld $\mathrm{MH}$, Compton SJ, Mead $\mathrm{RH}$, et al. Remote monitoring of implantable cardioverter defibrillators: a prospective analysis. Pacing Clin Electrophysiol 2004; $27(6$ Pt 1):757-63.

15. Santini M, Ricci RP, Lunati M, et al. Remote monitoring of patients with biventricular defibrillators through the CareLink system improves clinical management of arrhythmias and heart failure episodes. J Interv Card Electrophysiol 2009; 24:53-61.

16. Crossley GH, Chen J, Choucair W, et al; PREFER Study Investigators. Clinical benefits of remote versus transtelephonic monitoring of implanted pacemakers. J Am Coll Cardiol 2009; 54:2012-9.

17. Hauck M, Bauer A, Voss F, et al. "Home monitoring" for early detection of implantable cardioverter-defibrillator failure: a single-center prospective observational study. Clin Res Cardiol 2009; 98:19-24.

18. Spencker S, Coban N, Koch L, Schirdewan A, Müller D. Potential role of home monitoring to reduce inappropriate shocks in implantable cardioverter-defibrillator patients due to lead failure. Europace 2009; 11:483-8.

19. Vogtmann T, Stiller S, Marek A, et al. Workload and usefulness of daily, centralized home monitoring for patients treated with CIEDs: results of the MoniC (Model Project Monitor Centre) prospective multicentre study. Europace 2013; 15:219-26.

20. Ricci RP, Morichelli L, D'Onofrio A, et al. Effectiveness of remote monitoring of CIEDs in detection and treatment of clinical and devicerelated cardiovascular events in daily practice: the HomeGuide Registry. Europace 2013; 15:970-7.

21. Guédon-Moreau L, Lacroix D, Sadoul N, et al; ECOST trial Investigators. A randomized study of remote follow-up of implantable cardioverter defibrillators: safety and efficacy report of the ECOST trial. Eur Heart J 2013; 34:605-14.

22. Kallinen LM, Hauser RG, Tang C, et al. Lead integrity alert algorithm decreases inappropriate shocks in patients who have Sprint Fidelis pacesense conductor fractures. Heart Rhythm 2010; 7:1048-55.

23. Hauser RG, Hayes DL, Epstein AE, et al. Multicenter experience with failed and recalled implantable cardioverter-defibrillator pulse generators. Heart Rhythm 2006; 3:640-4.

24. Saxon LA, Hayes DL, Gilliam FR, et al. Long-term outcome after ICD and CRT implantation and influence of remote device follow-up: the ALTITUDE survival study. Circulation 2010; 122:2359-67.

25. Klersy C, De Silvestri A, Gabutti G, Regoli F, Auricchio A. A meta-analysis of remote monitoring of heart failure patients. J Am Coll Cardiol 2009; 54:1683-94

26. Raatikainen MJ, Uusimaa P, van Ginneken MM, Janssen JP, Linnaluoto M. Remote monitoring of implantable cardioverter defibrillator patients: a safe, time-saving, and cost-effective means for follow-up. Europace 2008; 10:1145-51.

27. Brugada $P$. What evidence do we have to replace in-hospital implantable cardioverter defibrillator follow-up? Clin Res Cardiol 2006; 95 Suppl 3:III3-9.

28. Theuns DA, Rivero-Ayerza M, Knops P, Res JC, Jordaens L. Analysis of 57,148 transmissions by remote monitoring of implantable cardioverter defibrillators. Pacing Clin Electrophysiol 2009; 32 Suppl 1:S63-5.

29. Halimi F, Clémenty J, Attuel P, Dessenne X, Amara W; OEDIPE trial Investigators. Optimized post-operative surveillance of permanent pacemakers by home monitoring: the OEDIPE trial. Europace 2008; 10:1392-9.

30. Ricci RP, Morichelli L, D'Onofrio A, et al. Manpower and outpatient clinic workload for remote monitoring of patients with cardiac implantable electronic devices: data from the HomeGuide Registry. J Cardiovasc Electrophysiol 2014; 25:1216-23.

31. Jung W, Rillig A, Birkemeyer R, Miljak T, Meyerfeldt U. Advances in remote monitoring of implantable pacemakers, cardioverter defibrillators and cardiac resynchronization therapy systems. J Interv Card Electrophysiol 2008; 23:73-85

32. Hernández-Madrid A, Lewalter T, Proclemer A, et al; Scientific Initiatives Committee; European Heart Rhythm Association. Remote monitoring of cardiac implantable electronic devices in Europe: results 
of the European Heart Rhythm Association survey. Europace 2014; 16:129-32

33. Ricci RP, Morichelli L, Quarta L, et al. Long-term patient acceptance of and satisfaction with implanted device remote monitoring. Europace 2010 12:674-9.

34. Department of Statistics Singapore. Yearbook of Statistics Singapore
2015. Available at: http://www.singstat.gov.sg/docs/default-source/ default-document-library/publications/publications_and_papers/reference/ yearbook_2015/yos2015.pdf. Accessed June 26, 2016.

35. Folino AF, Chiusso F, Zanotto G, et al. Management of alert messages in the remote monitoring of implantable cardioverter defibrillators and pacemakers: an Italian single-region study. Europace 2011; 13:1281-91. 